\title{
Catecholamine Effects Upon Rat Hypothalamic Corticotropin-releasing Hormone Secretion In Vitro
}

\author{
Aldo E. Calogero, William T. Gallucci, ${ }^{\star}$ George P. Chrousos, and Philip W. Gold* \\ Developmental Endocrinology Branch, National Institute of Child Health and Human Development, Bethesda, Maryland 20892; \\ and the *Biological Psychiatry Branch, National Institute of Mental Health, Bethesda, Maryland 20892
}

\begin{abstract}
To further our understanding of the functional role of catecholaminergic systems in regulating hypothalamic corticotropinreleasing hormone (CRH) secretion, we assessed the direct effects of a multiplicity of catecholamine agonists and antagonists on hypothalamic CRH secretion. To accomplish this, we used an in vitro rat hypothalamic organ culture system in which CRH secretion from single explants was evaluated by a specific RIA (IR-rCRH). Norepinephrine (NE) stimulated IRrCRH secretion dose dependently, with peak effects in the nanomolar range. The effect of $\mathrm{NE}$ was antagonized by the mixed $\alpha$ antagonist phentolamine, the $\alpha_{1}$ antagonist prazosin, and the $\alpha_{2}$ antagonist yohimbine, but not by the $\beta$ blocker, L-propanolol. Compatible with these data were the findings that the $\alpha_{1}$ agonist phenylephrine and the $\alpha_{2}$ agonist clonidine both stimulated IR-rCRH secretion in a dose-dependent fashion. On the other hand, whereas the $\beta$ agonist, isoproterenol, caused a weak, non-dose-dependent increase in IR-rCRH secretion, this effect could not be antagonized by L-propanolol. Despite pretreatment with serotonin and acetylcholine antagonists, the effect of NE upon IR-rCRH secretion was undiminished, suggesting that NE-induced CRH secretion is not mediated by either neurotransmitter. On the other hand, pretreatment with $\gamma$-aminobutyric acid (GABA) attenuated NE-induced IR-rCRH secretion. Whereas epinephrine (E) stimulated IR-rCRH secretion, this occurred only at higher concentrations, and was antagonized by phentolamine, but not by L-propanolol. Dopamine (DA) had a weak stimulatory effect that could be antagonized by the $\mathrm{DA}_{1}$ receptor antagonist, SCH 23390, but not by phentolamine.

We conclude that $\mathrm{NE}$ and $\mathrm{E}$ stimulate hypothalamic IRrCRH secretion via $\alpha_{1}$ and $\alpha_{2}$ receptors. The effect of NE upon IR-rCRH secretion is not apparently mediated by serotonergic or cholinergic interneurons, but is modulated by the inhibitory neurotransmitter, GABA. These data support the idea that the central catecholaminergic systems are excitatory rather than inhibitory upon CRH secretion when acting directly at the hypothalamic level.
\end{abstract}

\section{Introduction}

Catecholaminergic systems and especially the noredrenergic system have long been regarded as inhibitors of the hypotha-

Address reprint requests to Dr. Aldo E. Calogero, Developmental Endocrinology Branch, National Institute of Child Health and Development, Building 10, Room 10N262, National Institutes of Health, Bethesda, MD 20892.

Received for publication 8 September 1987 and in revised form 14 April 1988.

The Journal of Clinical Investigation, Inc.

Volume 82, September 1988, 839-846 lamic-pituitary-adrenal (HPA) ${ }^{1}$ axis (1-8). However, recent data obtained from experimental animals and humans indicate that the brainstem noradrenergic (i.e., locus coeruleus [LC]) system and the HPA axis are both activated during physical (e.g., surgery, trauma, hemorrhage, and exercise) and emotional (e.g., depression, panic anxiety attacks, bereavement, examinations, and experimentally induced inescapable shock stress) (9-25). These data suggest that catecholamines (CA) stimulate the HPA axis at the pituitary and/or hypothalamic/suprahypothalamic levels. There is general agreement that norepinephrine (NE) and epinephrine (E) are capable of stimulating pituitary ACTH in vitro (26) and that the stimulatory effect of both CA is additive to the stimulatory effect of corticotropin-releasing hormone $(\mathrm{CRH})(26,27)$.

On the other hand, both anatomical and functional studies indicate that CA also act at the hypothalamic site of the HPA axis. It has been shown that the parvocellular division of the paraventricular nucleus (PVN), which contains the majority of the CRH-positive perikarya in the hypothalamus (28-31), receives copious catecholaminergic innervations originating from the brainstem catecholaminergic regions (32-34). Furthermore, direct synaptic interactions between catecholaminergic terminals and CRH neurons have been demonstrated (35). Inferences from pharmacological studies regarding the functional nature (inhibitory or stimulatory) of these afferent noradrenergic fibers to the hypothalamic PVN remain, however, controversial. Many pharmacological studies have yielded data supporting a central inhibitory effect of CA on CRH secretion (36-40). Alternatively, other studies provide evidence for a stimulatory effect of CAs on the central component of the HPA axis (41-49).

This study was performed to evaluate the effects of a multiplicity of catecholamine agonists and antagonists on hypothalamic CRH secretion. To accomplish this, we used an in vitro hypothalamic organ culture system, in which immunoreactive rat CRH (IR-rCRH) secretion was measured directly from single explants. We also attempted to explore the potential interaction between NE and other neurotransmitters such as serotonin (5HT), acetylcholine, and gamma-aminobutyric acid (GABA) on CRH secretion.

1. Abbreviations used in this paper: ANOVA, analysis of variance; AVP, arginine vasopressin; CA, catecholamines; CR, corticotropin releasing; $\mathrm{CRH}$, corticotropin-releasing hormone; DA, dopamine; DHPG, 3,4 dihydroxyphenylethyleneglycol; E, epinephrine; 5HT, serotonin; GABA, gamma-aminobutyric acid; HPA, hypothalamic-pituitary-adrenal axis; ICV, intracerebroventricular; IR-rCRH, immunoreactive rat corticotropin-releasing hormone; $\mathrm{LC}$, locus coeruleus; MSH, melanocyte-stimulating hormone; NE, norepinephrine; QT, oxytocin; PVN, paraventricular nucleus; 6-OHDA, 6-hydroxydopamine; VIP, vasoactive intestinal peptide; VNAB, ventral noradrenergic ascending bundle. 


\section{Methods}

Materials. NE hydrochloride, E, clonidine hydrochloride, phenylephrine hydrochloride, isoproterenol hydrochloride, dopamine (DA), atropine methylbromide, hexamethonium bromide, and GABA were purchased from Sigma Chemical Co. (St. Louis, MO). SCH 23390 was purchased from Research Biomedicals; Inc. (Natick, MA). Phentolamine mesylate, prazosin hydrochloride, and ritanserin ( $R$ 55,667) were gifts from Ciba-Geigy Corp. (Summit, NJ), Pfizer Chemicals (Groton, CT), and Janssen Pharmaceutica (Piscataway, NJ), respectively.

Hypothalamic organ culture. Adult male Sprague-Dawley rats were decapitated. Using a sterile technique, we rapidly removed the hypothalami with fine-pointed curved scissors. The hypothalamic boundaries were the optic chiasm, anteriorly, the mammillary bodies, posteriorly, and the hypothalamic sulci, laterally. The fragments were $\sim 3 \mathrm{~mm}$ deep. Immediately after the explantation the hypothalami (one explant per incubation chamber, 48-multiwell plates, Costar Corp., MA) were incubated in $0.4 \mathrm{ml}$ medium 199 with modified Earle's salt (Gibco Laboratories, Grand Island, NY) containing $0.1 \%$ BSA (fraction V, Sigma Chemical Co.) and $20 \mu \mathrm{M}$ bacitracin (Aldrich Chemical Co., Milwaukee, WI), in a water-jacketed incubator at $37^{\circ} \mathrm{C}$, under 5\% $\mathrm{CO}_{2}$ atmosphere. The experiments, performed according to the protocols described below, were carried out after an overnight (18 h) preincubation. Detailed biological and histologic studies on freshly explanted and overnight preincubated hypothalami have been reported elsewhere. ${ }^{2}$ In brief, IR-rCRH content of hypothalami extracted after an overnight preincubation was not different from the IR-rCRH content of freshly explanted hypothalami. Histologic integrity of overnight-preincubated hypothalami was studied by both light and electron microscopy. Although the overnight-preincubated tissues exhibited a certain degree of swelling and were more fragile than the freshly excised tissues, the Nissl's staining of all sections was identical. In both sets of tissues, the PVN was distinguished by deeper staining and closer packing of cells than the surrounding territory, showing the typical features that characterize the nucleus. Cell dendrites were similar in both sets of explants. Ảt higher magnification, comparable intracellular staining heterogeneity was observed in the PVN neurons from freshly excised and overnight-preincubated tissues. The stain extended well into the proximal dendrites. Trypan blue was excluded from the cells of overnight-preincubated explant sections containing the PVN or median eminence.

By electron microscopy, PVN cells of freshly explanted hypothalami or overnight-preincubated tissues showed similar morphology. In both preparations, mitochondrial matrix and membrane and nuclear substance were preserved. The latter preparation showed some degree of demyelination, presumably a result denervation. No signs of cell necrosis were detected.

IR-rCRH secretion in plain medium, from a given hypothalamus incubated in the same well reached its plateau after $20 \mathrm{~min}$. A 20 -min incubation interval thus was chosen. The experimental design consisted of six serial passages of the hypothalamic explant in different wells, every $20 \mathrm{~min}$ for a total of $120 \mathrm{~min}$. During this time, the IR-rCRH secretion in control medium remained constant. The hypothalamic explants were transferred from well to well using a $3 \times 3 \mathrm{~mm}$ nylon mesh grid (Small Parts, Inc., Miami, FL). After each experiment (see Experimental Protocols below), we indirectly confirmed tissue viability by exposing each hypothalamus to a depolarizing concentration of $\mathrm{KCl}(60 \mathrm{mM})$ in the sixth well. Explants that failed to respond to $\mathrm{KCl}$ with an increase of IR-rCRH secretion of at least $90 \%$ (> 10 intraassay coefficients of variation) above baseline were excluded from

2. Calogero, A. E., R. Bernardini, A. N. Margioris, G. Bagdy, W. T. Gallucci, P. J. Munson, L. Tamarkin, T. P. Tomai, L. Brady, P. W. Gold, and G. P. Chrousos. 1988. Effects of serotonergic agonists and antagonists on corticotropin-releasing hormone secretion by explanted rat hypothalami. Manuscript submitted for publication. analysis. Lack of response to $\mathrm{KCl}$ was observed in $\sim 20 \%$ of the hypothalami used.

Experimental protocols. To determine the effects of NE, clonidine, phenylephrine, $\mathrm{E}$, isoproterenol, and DA, the hypothalami were incubated in plain medium in the first three wells (basal IR-rCRH concentration) and exposed to graded concentration of these substances in the next two wells (stimulated IR-rCRH concentration). Controls for this set of experiments were obtained exposing the hypothalami to medium with no test substance. To evaluate the effects of specific antagonists, the hypothalami were incubated in plain medium in the first two wells (basal IR-rCRH concentration). In the third, fourth, and fifth wells the antagonist(s) were present, whereas in the fourth and fifth wells the stimulant was added (stimulated IR-rCRH concentration). Finally, to determine the effect of GABA on NE-induced IR-rCRH secretion, GABA was added during the last hour of preincubation and throughout the experiment conducted according to protocol 1 .

$C R H$ radioimmunoassay. Medium concentration of $\mathrm{CRH}$ was measured directly without prior extraction by RIA, using a specific anti-rCRH serum (TS-3) obtained from rabbits immunized with synthetic rCRH conjugated to BSA. ${ }^{125} \mathrm{I}-\mathrm{rCRH}$, prepared by the chloramine $\mathrm{T}$ method (50), was stored at $4^{\circ} \mathrm{C}$ for up to $8 \mathrm{wk}$. An appropriate aliquot was purified through a $0.9 \times 58 \mathrm{~cm}$ column of Sephadex G-50 fine (Pharmacia Fine Chemicals, Piscataway, NJ), before use.

$100-\mu \mathrm{l}$ aliquots of $\mathrm{rCRH}$ standard solutions or media samples were incubated for $48 \mathrm{~h}$ at $4^{\circ} \mathrm{C}$ with TS-3 antiserum (final dilution 1:21,000 equilibrium association constant $=2.3 \times 10^{10} \mathrm{liter} / \mathrm{mol}$ ) diluted in assay buffer. After $48 \mathrm{~h}, 100 \mu \mathrm{l}$ of ${ }^{125} \mathrm{I}-\mathrm{rCRH}(3,000-3,200 \mathrm{cpm})$ were ádded and left to incubate for $24 \mathrm{~h}$. Separation of bound labeled rCRH from free hormone was achieved by incubating each tube for 12-16 h at $4^{\circ} \mathrm{C}$ with $50 \mu \mathrm{l}$ goat anti-rabbit serum, diluted $1: 5$ with assay buffer containing $1 \%$ BSA. The tubes were centrifuged at $2,000 \mathrm{~g}$ for $20 \mathrm{~min}$. The supernatants were aspirated and the precipitates were counted in a gamma-counter. Standards were measured in triplicate and samples in duplicate. Total binding of labeled rCRH by TS-3 antiserum was $33 \pm 3 \%($ mean $\pm \mathrm{SE})$ and nonspecific binding was $1.9 \pm 0.1 \%$. Sensitivity $\left(E D_{90}\right)$ of the assay was $2.0 \pm 0.1 \mathrm{pg} /$ assay tube $(20 \mathrm{pg} / \mathrm{ml}$ of media $)$. Parallelism to the standard curve was obtained with incubation medium containing CRH secreted by the hypothalami in vitro. The intraassay and interassay coefficients of variation were 7.9 and $15.6 \%$, respectively.

TS-3 antiserum showed no significant cross-reactivity with the following peptides: luteinizing hormone-releasing hormone, growth hormone-releasing hormone, thyrotropin-releasing hormone, somatostatin, substance $P$, porcine vasoactive intestinal peptide (VIP), neuropeptide Y, hACTH(1-39), $\beta$-endorphin, $\alpha$-melanocyte-stimulating hormone (MSH), $\beta$-MSH, dynorphin(1-17), arginine vasopressin (AVP), and oxytocin (OT).

Analysis of data. The results (mean $\pm \mathrm{SE}$ ) were calculated as percent IR-rCRH increase above baseline for each hypothalamus, applying the following formula. $\triangle \mathrm{CRH}(\%)=$ (stimulated IR - rCRH secretion/ basal IR - rCRH secretion - 1) $\times 100$. Statistical analysis was performed using one-way analysis of variance (ANOVA) followed by Duncan's multiple range test (51). Logarithmic transformation of the data was applied to correct for heteroscedasticity, detected by the Bartlett test (52).

\section{Results}

Basal IR-rCRH secretion in plain medium was $48.3 \pm 5.7 \mathrm{pg} /$ hypothalamus per $0.4 \mathrm{ml}$ per $20 \mathrm{~min}$, whereas mean $\pm \mathrm{SE}$ basal IR-rCRH secretion of all the experiments performed for this study was $49.3 \pm 5.3 \mathrm{pg} / \mathrm{hypothalamus}$ per $0.4 \mathrm{ml}$ per $20 \mathrm{~min}$ ( $n$ $=33$ experiments, $m=432$ measurements). NE stimulated IR-rCRH release in a dose-dependent fashion (Fig. 1). $10^{-12} \mathrm{M}$ NE was the lowest concentration capable of producing a significant IR-rCRH secretion, $49 \pm 39 \%$ above baseline $(P<0.05$ vs. 


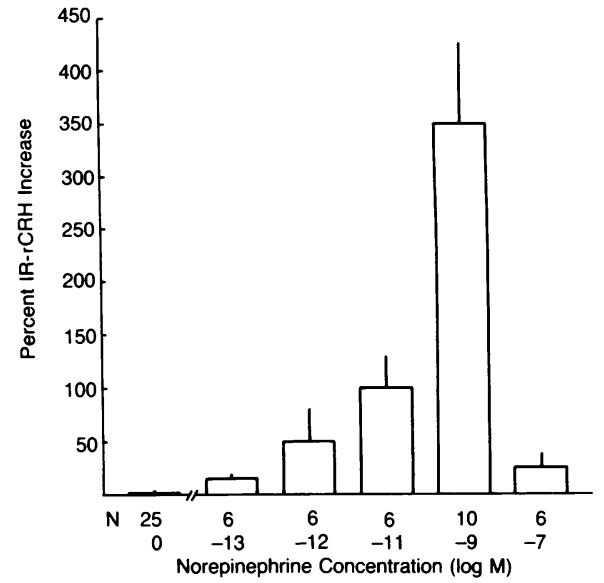

Figure 1. IR-rCRH secretion by single explanted hypothalami exposed to graded concentrations of NE. Results (mean \pm SE) are expressed as percent increase above baseline. NE induced a dose-dependent increase of IR-rCRH secretion. $\mathrm{N}=$ number of hypothalami tested.

concentration zero; Duncan test). Peak of IR-rCRH response (349 $\pm 85 \%$ increase above baseline) was obtained with an NE concentration of $10^{-9} \mathrm{M}(P<0.05$ vs. all the other concentrations tested; Duncan test). The highest NE concentration tested $\left(10^{-7} \mathrm{M}\right)$ induced only $18 \pm 17 \%$ IR-rCRH increase above baseline ( $P<0.05$ vs. concentration zero; Duncan test).

To explore the adrenergic receptor types involved in the stimulatory action of NE upon hypothalamic CRH secretion, we examined the effect of a variety of pharmacologic antagonists on NE- $\left(10^{-9} \mathrm{M}\right)$ induced IR-rCRH secretion (Fig. 2). Phentolamine, an $\alpha_{1}$ and $\alpha_{2}$ receptor antagonist, profoundly antagonized NE-induced IR-rCRH release at both $\left(10^{-9}\right.$ and $10^{-7} \mathrm{M}$ ) concentrations used $60 \pm 19$ and $14 \pm 11 \%$, above baseline, respectively, vs. $349 \pm 85 \% ; P<0.05$ for both concentra-

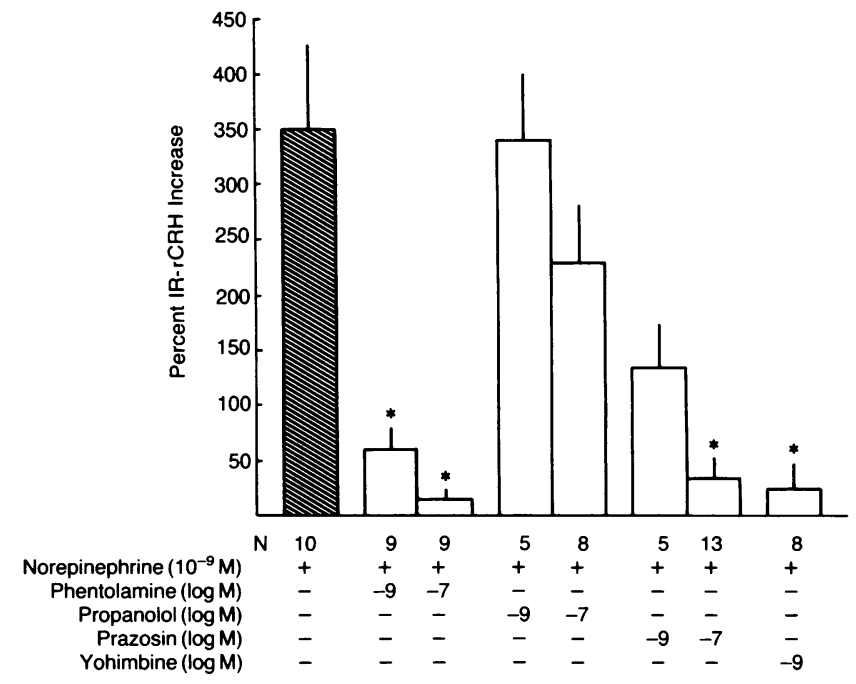

Figure 2. IR-rCRH secretion by single explanted hypothalami exposed to $10^{-9} \mathrm{M} \mathrm{NE}$ (shaded bar) or NE plus different concentrations of noradrenergic antagonists. None of these antagonists had any effect on basal IR-rCRH secretion. Results (mean \pm SE) are expressed as percent increase above baseline. ${ }^{*} P<0.05$ vs. NE alone; Duncan test. $\mathbf{N}=$ number of hypothalami tested.

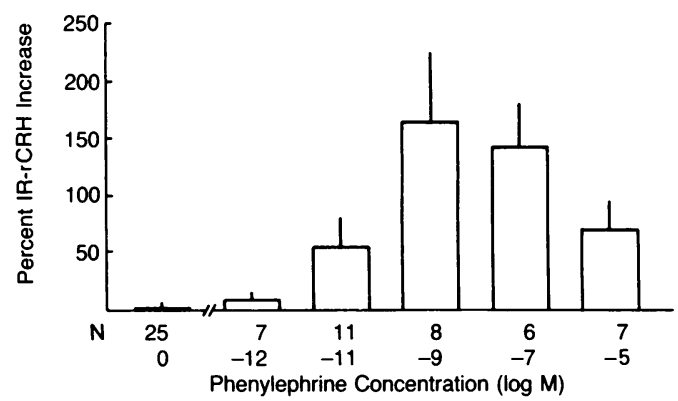

B

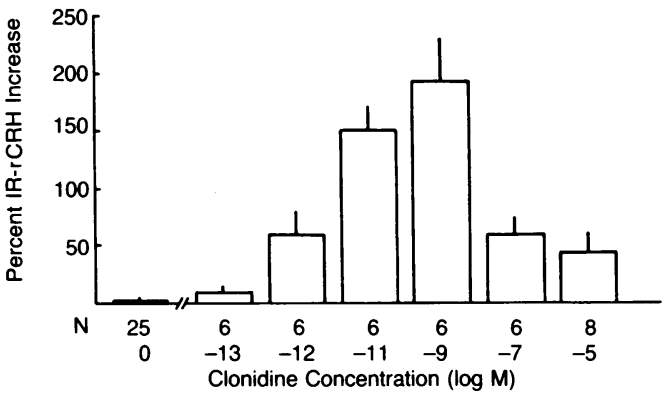

Figure 3. IR-rCRH secretion by single explanted hypothalami exposed to graded concentrations of phenylephrine $(A)$ or clonidine $(B)$. Results (mean $\pm \mathrm{SE})$ are expressed as percent increase above baseline. Both phenylephrine and clonidine induced a dose-dependent increase of IR-rCRH secretion. $\mathrm{N}=$ number of hypothalami tested.

tions; Duncan test). Propanolol, a $\beta_{1}$ and $\beta_{2}$ receptor antagonist, had no effect on NE-induced IR-rCRH secretion. Prazosin, a specific $\alpha_{1}$ antagonist, was able to antagonize NE-induced IR-rCRH only at the highest concentration used $\left(10^{-7} \mathrm{M} ; 35 \pm 20\right.$ vs. $349 \pm 85 \%$ above baseline; $(P<0.05$ vs. $\mathrm{NE}$ alone; Duncan test). Yohimbine, an $\alpha_{2}$ receptor antagonist, at concentration of $10^{-9} \mathrm{M}$, also significantly inhibited NE-induced IR-rCRH secretion $(P<0.05$ vs. NE alone; Duncan test).

We also assessed the effects of pharmacologic agonists of adrenergic receptor subtypes (Figs. 3 and 4). Phenylephrine, an $\alpha_{1}$ adrenergic agonist, stimulated IR-rCRH secretion in a dose-dependent fashion (Fig. $3 \mathrm{~A}$ ). The lowest stimulatory concentration $\left(10^{-11} \mathrm{M}\right)$ induced an IR-rCRH rise of $55 \pm 24 \%$ above baseline ( $P<0.05$ vs. concentration zero; Duncan test). Clonidine, an $\alpha_{2}$ receptor agonist (Fig. $3 \mathrm{~B}$ ), also stimulated IR-rCRH secretion in a dose-dependent fashion, with $10^{-12} \mathrm{M}$ being the lowest stimulatory concentration $(60 \pm 21 \%$ increase above baseline; $P<0.05$ vs. concentration zero; Duncan test). The greater potency of clonidine in inducing IR-rCRH secretion compared with the $\alpha_{1}$ agonist phenylephrine, is compatible with the greater antagonism of $\alpha_{2}$ versus $\alpha_{1}$ antagonists. Isoproterenol, a $\beta_{1}$ and $\beta_{2}$ agonist, had a weak, non-dose-dependent stimulatory effect upon IR-rCRH secretion (Fig. 4). This stimulatory action of isoproterenol could not be antagonized by propanolol or phentolamine, suggesting a nonspecific rather than receptor-mediated mechanism (Fig. 4, inset).

To determine whether other neurotransmitter systems modulated the $\alpha$-adrenergic-mediated NE-induced IR-CRH secretion, we assessed the effects of antagonists to 5HT type 2 (ritanserin), muscarinic (atropine), and nicotinic (hexamethonium) receptors. We also assessed the effect of the inhibitory neurotransmitter GABA upon NE-induced IR-CRH secretion (Fig. 5). Neither ritanserin nor atropine and hexamethonium, 


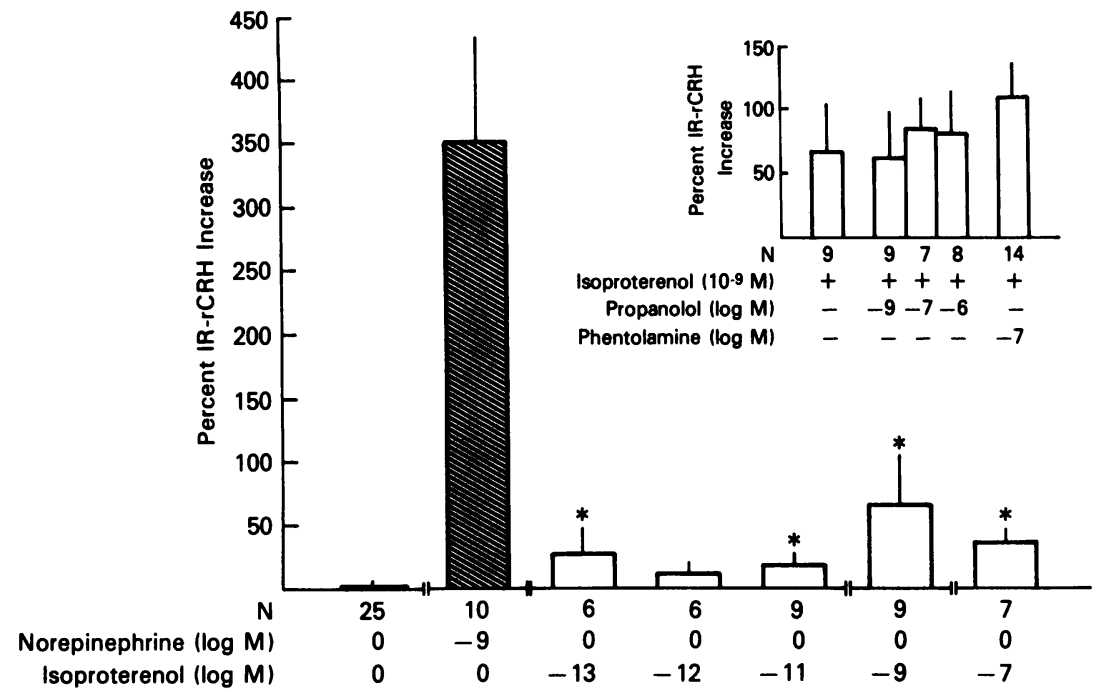

Figure 4. IR-rCRH secretion by single explanted rat hypothalami exposed to graded concentrations of isoproterenol and $10^{-9} \mathrm{M} \mathrm{NE}$ as positive control (shaded bar). Results (mean \pm SE) are expressed as percent increase above baseline. ${ }^{*} P$ $<0.05$ vs. concentration zero; Duncan test. (Inset) IR-rCRH secretion by single explanted hypothalami (mean $\pm \mathrm{SE}$ ) exposed to $10^{-9} \mathrm{M}$ isoproterenol or isoproterenol plus increasing concentrations of propanolol or isoproterenol plus phentolamine. $\mathbf{N}=$ number of hypothalami tested. all used at $10^{-7} \mathrm{M}$ (a concentration known to antagonize 5HTand acetylcholine-induced IR-rCRH secretion in this system [see footnote 2, reference 53]), influenced NE-induced IRrCRH secretion. Whereas, GABA at $10^{-7} \mathrm{M}$, but not at a concentration of $10^{-9} \mathrm{M}$ was able to inhibit this parameter ( $P$ $<0.05$ vs. NE alone; Duncan test).

We also determined the effects of other catecholamines; namely, E and DA, on in vitro IR-rCRH secretion. E stimulated IR-rCRH secretion in a dose-dependent fashion (Fig. 6). $10^{-11} \mathrm{M}$ E was the minimal stimulatory concentration ( $P$ $<0.05$ vs. concentration zero; Duncan test). Maximal stimulatory effect of $E$ upon IR-rCRH secretion was obtained at concentrations of $10^{-9}$ and $10^{-7} \mathrm{M}\left(P<0.05\right.$ vs. $10^{-11} \mathrm{M}$ concen-

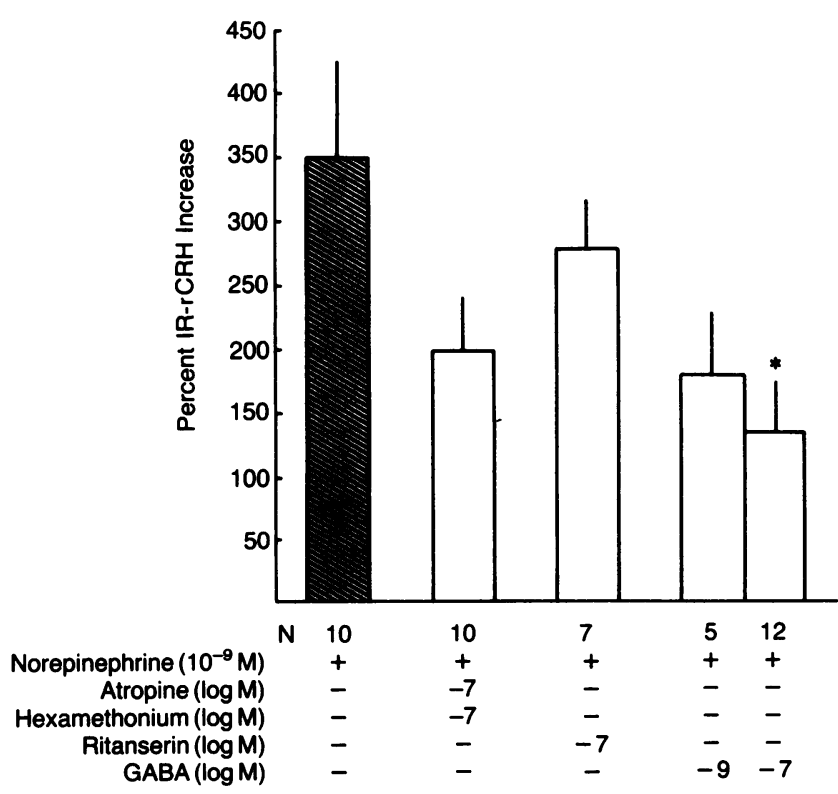

Figure 5. IR-rCRH secretion by single explanted hypothalami exposed to $10^{-9} \mathrm{M} N E$ (shaded bar) or NE plus cholinergic antagonists (atropine and hexamethonium), NE plus a 5HT type 2 receptor antagonist (ritanserin), and NE plus GABA. Results (mean \pm SE) are expressed as percent increase above baseline. ${ }^{*} P<0.05$ vs. norepinephrine alone; Duncan test. $\mathbf{N}=$ number of hypothalami tested. tration; Duncan test). A $10^{-5} \mathrm{M}$ concentration of epinephrine was unable to stimulate IR-rCRH secretion ( $4 \pm 3 \%$ increase above baseline vs. $3.4 \pm 1.3 \%$ increase induced by the control). E- $\left(10^{-9} \mathrm{M}\right)$ induced IR-rCRH secretion was antagonized by phentolamine $(P<0.05$ vs. $\mathrm{E}$ alone for both concentrations used; Duncan test), but not by $10^{-7} \mathrm{M}$ propanolol, (Fig. 6, inset).

DA had a weak stimulatory effect on IR-rCRH secretion (Fig. 7; $P<0.001$; ANOVA). The effect of $10^{-9} \mathrm{M}$ DA could be antagonized by the compound $\mathrm{SCH} 23390$, a $\mathrm{DA}_{1}$ receptor antagonist, $75 \pm 20$ vs. $14 \pm 12 \%$ increase above baseline $(P$ $<0.05$; Duncan test), but not by phentolamine (Fig. 7 , inset), suggesting that the stimulatory effect of DA upon IR-rCRH 


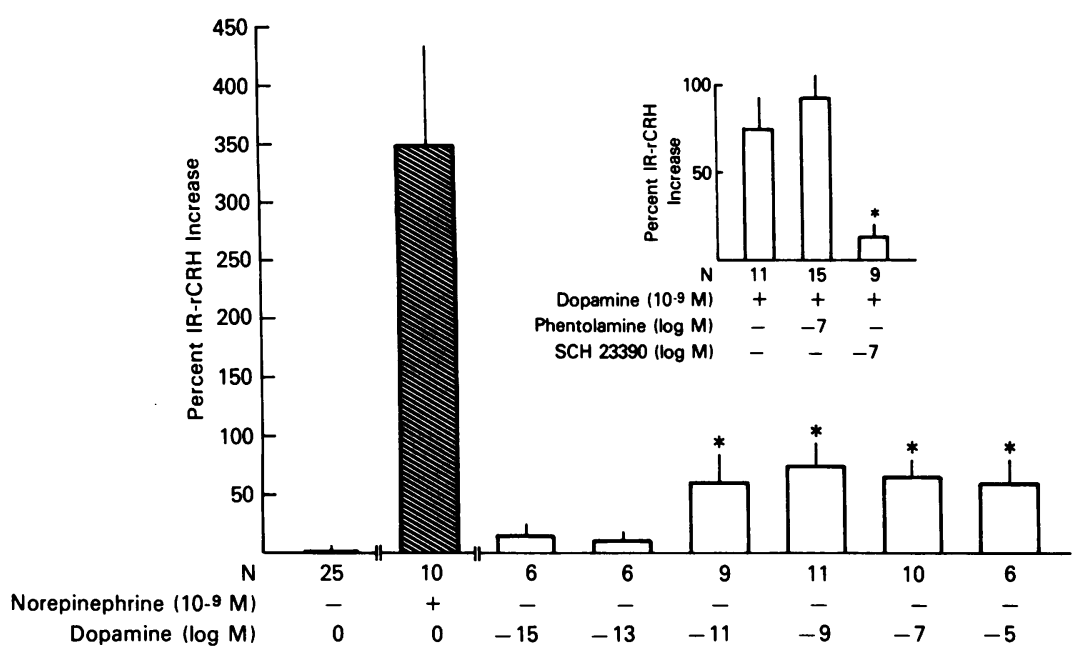

Figure 7. IR-rCRH secretion by single explanted hypothalami exposed to graded concentrations of $\mathrm{DA}$ and $10^{-9} \mathrm{M} \mathrm{NE}$ as positive control (shaded bar). Results (mean $\pm \mathrm{SE})$ are expressed as percent increase above baseline. ${ }^{*} P<0.05$ vs. concentration zero; Duncan test. $\mathrm{N}=$ number of hypothalami tested. (Inset) IR-rCRH secretion by single explanted hypothalami exposed to $10^{-9} \mathrm{M} \mathrm{DA}$, DA plus phentolamine, DA plus SCH $23390 .{ }^{*} P$ $<0.05$ vs. DA alone; Duncan test. $\mathrm{N}=$ number of hypothalami tested. secretion was receptor mediated and not due to the conversion of this neurotransmitter into NE or $\mathrm{E}$.

\section{Discussion}

The results of this study suggest that NE stimulates hypothalamic CRH secretion via $\alpha_{1}$ and $\alpha_{2}$ receptor activation. Hence, the $\mathrm{CRH}$ response to a maximal stimulatory concentration of NE was significantly antagonized by both $\alpha_{1}$ and $\alpha_{2}$ receptor antagonists. Moreover, both $\alpha_{1}$ and $\alpha_{2}$ agonists caused a significant increase in CRH secretion that was similar to, but less pronounced than that caused by NE. Although isoproterenol, a specific $\beta$ agonist, stimulated hypothalamic $\mathrm{CRH}$ secretion, its effect did not appear to be receptor mediated, since a specific $\beta$-adrenergic antagonist could not antagonize it. Therefore $\beta$-adrenergic receptors apparently are not involved in the stimulatory effect of NE upon in vitro hypothalamic CRH. The lack of inhibition of NE-induced CRH secretion by serotonergic (ritanserin) or cholinergic (atropine and hexamethonium) antagonists indicates that serotonergic or cholinergic interneurons do not mediate the stimulatory effect of NE on hypothalamic CRH secretion, whereas GABA apparently modulates NE action upon hypothalamic CRH secretion in the rat. Epinephrine stimulated IR-rCRH secretion less markedly (dose-response curve shifted to the right) than NE, whereas DA had a weak stimulatory effect upon hypothalamic CRH secretion. The effect of DA did not appear to be due to the in vitro conversion of DA into other CAs, because phentolamine at concentrations capable of antagonizing NE-induced IR-rCRH secretion, did not have any effect on dopamine-induced IR-rCRH secretion. On the other hand, the DA stimulatory effect was antagonized by $\mathrm{DA}_{1}$ receptor blockade. The stimulatory effect of DA on hypothalamic $\mathrm{CRH}$ secretion thus apparently proceeds via $\mathrm{DA}_{1}$ receptor activation.

Despite the prolonged incubation period used in this study, several lines of evidence indicate intactness of the hypothalamic explants upon which our data are based. Hence, compared with freshly explanted hypothalami, the explants used in this design showed similar CRH content, similar capacity to exclude trypan blue, histologic integrity of PVN cells and fibers projecting to the median eminence by light microscopy, and an electron microscopic appearance showing intactness of subcellular fractions in the context of edema and a small amount of fiber demyelination (see footnote 2). Note that our hypothalamic explants represent deafferented central nervous system tissue. A time-dependent increase in sensitivity to regulatory stimuli is expected from such a tissue. This sensitivity change could enhance the capacity of an in vitro system to yield information regarding the regulation of a neuromodulator like CRH, although the concentrations required to produce responses in this system may be lower than those required to produce the same effect in vivo. However, we can not rule out the possibility that reversal of responsiveness to regulatory agents might occur under the conditions used here, although to our knowledge, this has not been previously reported for hypothalamic tissue.

Catecholamines have long been regarded as inhibitors of the HPA axis (1-8). A number of studies in anesthetized dogs and rats have demonstrated that the intracerebroventricular (ICV) administration of NE or NE agonists reduces adrenocortical secretion $(2,6,7)$ whereas the systemic or ICV administration of NE antagonists activates the HPA axis $(3,4)$. Furthermore, Rose et al. showed that in anesthetized dogs, electrical stimulation of the ventral noradrenergic ascending bundle (VNAB), which includes almost all the catecholaminergic pathways to the PVN, inhibits stress-induced (laparotomy) ACTH secretion (5). Additional in vivo data supporting an inhibitory role for NE on HPA axis function is the finding that the $\alpha_{2}$ agonist clonidine decreases plasma ACTH and cortisol secretion in human subjects (8). It is possible, however, that $\mathrm{NE}$ and NE agonists and antagonists, systemically or ICV injected, may act at several potential sites within the brain (i.e., LC or PVN) and in the periphery (i.e., pituitary) to influence HPA axis function $(26,54)$. In this regard, Weiss et al. have shown that severely stressed rats, who invariably show a marked increase of their LC firing rates, respond to the systemic, ICV, and local administration of NE or NE agonists (e.g., monoamine oxidase inhibitors and tricyclic antidepressants) with a decrease in the LC firing rate (19). Weiss proposed that this effect of $\mathrm{NE}$ agonists on $\mathrm{LC}$ firing rates was mediated by an interaction between NE and presynaptic $\alpha_{2}$ inhibitory receptors in the region of the LC. The latter are the only adrenergic receptors known to reside in this area (55). Hence, the ICV NE agonist-induced decrease of adrenocortical activity could be mediated by inhibition of LC-mediated stimulation of the PVN (54). Conversely, the systemic or ICV administration of NE antagonists could increase adrenocortical function by enhancing LC activation of hypothalamic CRH 
neurons (56). Another possible factor to be considered in interpreting the findings of these studies is the use of anesthetized animals. It is known that anesthetic agents can change the magnitude and/or direction of central nervous system response to physical, chemical, or electrical stimuli $(15,57)$. All of these studies also assessed hypothalamic CRH secretion indirectly, by measuring plasma ACTH and/or glucocorticoid concentrations as endpoints.

Inhibitory effects of CAs on the HPA axis also have been suggested on the basis of in vitro studies performed with hypothalamic explants (36-40). However, most of these studies (36-39) measured corticotropin-releasing (CR) bioactivity rather than CRH as endpoint and it is now known that several hypothalamic factors exert CR bioactivity. These include AVP, OT, 5HT, E, and VIP (58). Only one prior study, conducted by Suda et al., used direct measurements of immunoreactive CRH in exploring the direct effects of NE upon the CRH neuron (40). NE had an inhibitory effect on CRH secretion mediated by $\beta$, and to a lesser extent, by $\alpha_{1}$ adrenergic receptor mechanisms. Note the finding of previous studies describing paucity of $\beta$ adrenergic receptors in the hypothalamus (59). Four major differences in design should be noted between the Suda study and ours. First, the study of Suda et al. did not attempt to ascertain that hypothalami included in the study sustained no damage during the experimental procedure. Second, it failed to include the kind of comprehensive data shown here, in which responses to a large series of catecholamine agonists and antagonists provide consistent and corroborating results. Third, Suda et al, incubated two hypothalami per incubation chamber, introducing the added complexities of interactions between the two different units, the possibility of differencial necrosis, etcetera. Suda et al. also used a much shorter preincubation period than that used in our study. Hence, the explants used may not have recovered from the influence of neurotransmitters and hormones secreted in great amounts during the stress of decapitation for explantation. In this regard we have found that glucocorticoids, products of the proopiomelanocortin gene (ACTH, $\beta$ endorphin, $\alpha$-MSH), and CRH itself are all able to suppress CRH secretion in vitro (60). We have found that $5 \mathrm{HT}^{2}$ and acetylcholine (53), which were both excitatory to CRH secretion under conditions identical to those of this study, failed to release CRH from hypothalami exposed to a short preincubation period.

In comparison to the above studies and in corroboration of our data, a number of in vitro and in vivo studies have suggested that NE stimulates CRH secretion (41-49). Using an in vitro hypothalamic organ culture system and pituitary ACTH secretion as CRH index, Fehm and collaborators demonstrated that activation of $\alpha$ adrenergic receptors stimulates hypothalamic CR bioactivity in a dose-dependent fashion. Although their bell-shaped dose-response curve was similar to ours, it was shifted to the right, with the peak response occurring at $\sim 10^{-6} \mathrm{M}$ concentration (41).

Several in vivo studies have also shown a stimulatory effect of NE on the central component of the HPA axis. Krieger and Krieger showed that implantation of NE directly into the hypothalamus of the cat increases $11-\mathrm{OH}$ corticosteroid secretion in the awake state (42). In the rat, Smythe et al. showed an increase in the 3,4-dihydroxyphenylethyleneglycol (DHPG)/ $\mathrm{NE}$ ratio (indicative of increased NE neuronal activity) after cold water swim stress, ether stress, and adrenalectomy, and a positive correlation between the hypothalamic DHPG/NE ratio and basal pituitary-adrenal function (43). In a similar vein, Roy et al. have shown that there is a positive correlation between cerebrospinal fluid concentrations of CRH and NE in humans (23). In a different approach, Szafarczyk et al. have demonstrated that injection of the catecholaminergic toxin 6-hydroxydopamine (6-OHDA) into the rat VNAB profoundly diminished the plasma ACTH response to stressful stimuli and virtually abolished basal circadian ACTH secretion (45). More recently, this same group has shown that ICV injected NE and E induced a stress-like ACTH surge in either intact rats or animals that were previously treated with 6OHDA. This CA-induced ACTH stimulation was prevented by immunoneutralization with anti-CRH serum (46). In addition, Guillaume et al. have shown that 6-OHDA-induced destruction of the VNAB reduced the levels of immunoreactive CRH in the rat hypophysial portal blood (47) and Plotsky has shown that either electrical activation of central endogenous catecholaminergic pathways or ICV injection of NE increased hypophysial portal blood levels of IR-rCRH via $\alpha_{1}$, but not via $\beta$-adrenergic receptors (48). In corroboration of the above findings, Damluji et al. clearly showed that central $\alpha_{1}$ adrenergic stimulation resulted in secretion of ACTH in man, presumably by causing release of CRH (49).

In conclusion, our hypothalamic organ culture system has allowed us to study the effects of many catecholamine agonists and antagonists upon hypothalamic CRH secretion. Although our experimental model used deafferented and relatively longterm incubated hypothalami, catecholamines and norepinephrine in particular, reproducibly stimulated CRH secretion. A possible in vivo stimulatory NE effect on hypothalamic CRH secretion may be of interest in light of recent data showing that CRH administration to awake, unrestrained rats markedly increases the LC firing rate $(61,62)$. These data, taken together, suggest that hypothalamic CRH neurons and NE neurons in regions such as the LC may participate in a mutually reinforcing positive feedback loop. These observations may be pertinent to stressful situations and psychiatric conditions, like depression and panic disorder, characterized by activation of both the LC-NE and the CRH systems (63).

\section{References}

1. Van Loon, G. R., U. Scapagnini, G. P. Moberg, and W. F. Ganong. 1971. Evidence for central adrenergic neural inhibition of ACTH secretion in the rat. Endocrinology. 89:1464-1469.

2. Van Loon, G. R., U. Scapagnini, R. Cohen, and W. F. Ganong. 1971. Effect of intraventricular administration of adrenergic drugs on the adrenal venous 17-hydroxycorticosteroid response to surgical stress in the dog. Neuroendocrinology. 8:257-272.

3. Scapagnini, U., G. R. Van Loon, G. P. Moberg, P. Preziosi, and W. F. Ganong. 1972. Evidence for central norepinephrine-mediated inhibition of ACTH secretion in the rat. Neuroendocrinology. 10:155160.

4. Eisenberg, R. M. 1975. Further evidence of a central $\alpha$-adrenergic inhibitory influence of the hypothalamo-pituitary-adrenal axis in the rat. Neuroendocrinology. 17:154-166.

5. Rose, J. C., P. C. Goldsmith, F. J. Holland, S. L. Kaplan, and W. F. Ganong. 1976. Effect of electrical stimulation of the canine brain stem on the secretion of ACTH and growth hormone (GH). Neuroendocrinology. 22:352-362.

6. Ganong, W. F., N. Kramer, J. Salmon, I. A. Reid, R. Lovinger, U. Scapagnini, A. T. Boryczka, and R. Shackelford. 1976. Pharmaco- 
logical evidence for inhibition of ACTH secretion by central noradrenergic system in the dog. Neuroscience. 1:167-174.

7. Ganong, W. F. 1980. Neurotransmitters and pituitary function: regulation of ACTH secretion. Fed. Proc. 39:2923-2930.

8. Lanes, R., A. Herrera, A. Palacios, and G. Moncada. 1983. Decreased secretion of cortisol and ACTH after oral clonidine administration in normal adults. Metab. Clin. Exp. 32:568-570.

9. Plotsky, P. M. 1988. Hypophysiotropic regulation of stress-induced ACTH secretion. In Physical and Emotional Stress: Mechanisms and Clinical Implications. G. P. Chrousos, D. L. Loriaux, and P. W. Gold, editors. Plenum Publishing Corp., New York. In press.

10. Axelrod, J., and T. D. Reisine. 1984. Stress hormones: their interaction and regulation. Science (Wash. DC). 224:452-459.

11. Axelrod, J., R. A. Muller, J. P. Henry, and P. M. Stephens. 1970. Changes in enzymes involved in the biosynthesis and metabolism of noradrenaline and adrenaline after psychosocial stimulation. Nature (Lond.). 225:1059-1060.

12. Henry, J. P., P. M. Stephens, J. Axelrod, and R. A. Muller. 1971. Effect of psychosocial stimulation on the enzymes involved in the biosynthesis and metabolism of noradrenaline and adrenaline. Psychosom. Med. 33:227-237.

13. Kvetnansky, R., and L. Mikulaj. 1970. Adrenal urinary catecholamines in rats during adaptation to repeated immobilization stress. Endocrinology. 87:738-743.

14. Kvetnansky, R., C. L. Sun, C. R. Lake, N. Thoa, T. Torda, and I. J. Kopin. 1978. Effects of handling and forced immobilization on rat plasma levels of epinephrine, norepinephrine, and dopamine- $\beta$-hydroxylase. Endocrinology. 103:1868-1874.

15. Udelsman, R., J. A. Norton, S. E. Jelenich, D. S. Goldstein, W. M. Linehan, D. L. Loriaux, and G. P. Chrousos. 1987. Responses of the hypothalamic-pituitary-adrenal and renin-angiotensin axes and the sympathetic system during controlled surgical and anesthetic stress. J. Clin. Endocrinol. \& Metab. 64:986-994.

16. Plotsky, P. M., and W. Vale. 1984. Hemorrhage-induced secretion of corticotropin-releasing factor-like immunoreactivity into the rat hypophysial portal circulation and its inhibition by glucocorticoids. Endocrinology. 114:164-169.

17. Luger, A., P. A. Duester, S. B. Kyle, W. T. Gallucci, L. C. Montgomery, P. W. Gold, D. L. Loriaux, and G. P. Chrousos. 1987. Acute hypothalamic-pituitary-adrenal responses to the stress of treadmill exercise: physiologic adaptations to physical training. $N$. Engl. J. Med. 316:1309-1315.

18. Christensen, N. J., and O. Brandsborg. 1973. The relationship between plasma catecholamine concentration and pulse rate during exercise and standing. Eur. J. Clin. Invest. 3:299-306.

19. Weiss, J. M., and P. G. Simson. 1985. Depression in an animal model: focus on the locus ceruleus. Ciba Found. Symp. 123:191-215.

20. Koslow, J. H., J. W. Maas, C. L. Bowden, J. M. Davis, I. Hanin, and J. Javaid. 1983. CSF and urinary biogenic amines and metabolites in depression and mania. Arch. Gen. Psychiatry. 40:999-1010.

21. Lake, C. R., D. Pickar, M. Ziegler, S. Slater, and D. L. Murphy. 1982. High plasma norepinephrine levels in patients with major affective disorders. Am. J. Psychiatry. 139:1315-1318.

22. Gold, P. W., D. L. Loriaux, A. Roy, M. A. Kling, J. R. Calabrese, C. H. Kellner, L. K. Nieman, R. M. Post, D. Pickar, W. Gallucci, P. Avgerinos, S. Paul, E. H. Oldfield, G. B. Cutler, and G. P. Chrousos. 1986. Responses to corticotropin-releasing hormone in the hypercortisolism of depression and Cushing's disease: pathophysiologic and diagnostic implications. N. Engl. J. Med. 314:1329-1335.

23. Roy, A., D. Pickar, M. Linnoila, G. P. Chrousos, and P. W. Gold. 1987. Cerebrospinal fluid corticotropin-releasing hormone in depression: relation to noradrenergic functions. Psychiatric Res. 20:229-237.

24. Uhde, T. W., J. P. Boulenger, R. M. Post, L. J. Siever, B. J. Vittone, D. C. Jimerson, and P. P. Roy-Byrne. 1984. Fear and anxiety: relationship to noradrenergic function. Psychopathology. 17(Suppl. 3):8-23.

25. Roy-Byrne, P. P., T. W. Udhe, R. M. Post, W. Gallucci, G. P.
Chrousos, and P. W. Gold. 1986. Corticotropin-releasing hormone stimulation test in patients with panic disorder. Am. J. Psychiatry. 143:896-899.

26. Vale, W., J. Vaughan, M. Smith, G. Yamamoto, J. Rivier, and C. Rivier. 1983. Effects of synthetic ovine corticotropin-releasing factor, glucocorticoids, catecholamines, neurohypophysial peptides, and other substances on cultured corticotropic cells. Endocrinology. 113:1121-1131.

27. Murakami, K., K. Hashimoto, and Z. Ota. 1984. Effect of angiotensin II, catecholamines and glucocorticoid on corticotropin-releasing factor (CRF)-induced ACTH release in pituitary cell cultures. Acta Med. Okayama. 38:349-355.

28. Weigand, S. J., and J. L. Price. 1980. The cells of origin of afferent fibers to the median eminence in the rat. J. Comp. Neurol. 192:1-19.

29. Makara, G. B., E. Stark, M. Karteszi, M. Palkovits, and G. Rappay. 1981. Effects of paraventricular lesions on stimulated ACTH release and CRF in stalk-median eminence of the rat. Am. J. Physiol. 240:E441-E446.

30. Antoni, F. A., M. Palkovits, G. B. Makara, E. A. Linton, P. J. Lowry, and J. Z. Kiss. 1983. Immunoreactive corticotropin-releasing hormone in the hypothalamo-infundibular tract. Neuroendocrinology. $36: 415-432$.

31. Sawchenko, P. E., and L. W. Swanson. 1985. Localization, colocalization, and plasticity of corticotropin-releasing factor immunoreactivity in the rat brain. Fed. Proc. 44:221-227.

32. Ungerstedt, U. 1971. Stereotaxic mapping of the monoamine pathways in the rat brain. Acta Physiol. Scand. Suppl. 367:1-48.

33. Lindvall, O., and A. Bjorklund. 1974. The organization of ascending catecholamine neuron systems in the rat brain. Acta Physiol. Scand. Suppl. 412:1-48.

34. Sawchenko, P. E., and L. W. Swanson. 1981. Central noradrenergic pathways for the integration of hypothalamic neuroendocrine and autonomic responses. Science (Wash. DC). 214:685-687.

35. Liposits, Zs., C. Phelix, and W. K. Paull. 1986. Electron microscopic analysis of tyrosine hydroxylase, dopamine- $\beta$-hydroxylase and phenylethanolamine- $N$-methyltransferease immunoreactive innervation of the hypothalamic paraventricular nucleus in the rat. Histochemistry. 84:105-120.

36. Hillhouse, E. W., J. Burden, and M. T. Jones. 1975. The effect of various putative neurotransmitters on the release of corticotropinreleasing hormone from the hypothalamus of the rat in vitro. I. The effect of acetylcholine and noradrenaline. Neuroendocrinology. 17:1-11.

37. Jones, M. T., and E. W. Hillhouse. 1977. Neurotransmitter regulation of corticotropin-releasing factor in vitro. Ann. NY Acad. Sci. 297:536-560.

38. Buckingham, J. C., and J. R. Hodges. 1979. Hypothalamic receptors influencing the secretion of corticotropin releasing hormone in the rat. J. Physiol. 290:421-431.

39. Jones, M. T., B. Gillham, B. D. Greenstein, R. R. Abraham, A. Dornhorst, U. Beckford, M. C. Holmes, J. H. Lin, A. Torrellas, N. G. Bowery, G. Di Renzo, and F. Knowles. 1982. The role of neurotransmitters and glucocorticoid hormones in the control of adrenocorticotrophin secretion. In Pituitary Hormones and Related Peptides. M. Motta, M. Zanisi, and F. Piva, editors. Academic Press Inc., New York. 49:281-303.

40. Suda, T., F. Yajima, N. Tomori, T. Sumitomo, Y. Nakagami, T. Ushiyama, H. Demura, and K. Shizume. 1987. Inhibitory effect of norepinephrine on immunoreactive corticotropin-releasing factor from the rat hypothalamus in vitro. Life Sci. 40:1645-1649.

41. Fehm, H. L., K. H. Voigt, R. E. Lang, and E. F. Pfeiffer. 1980. Effects of neurotransmitters on the release of corticotropin releasing hormone (CRH) by rat hypothalamic tissue in vitro. Exp. Brain Res. 39:229-234.

42. Krieger, H. P., and D. T. Krieger. 1970. Chemical stimulation of the brain: effect on adrenal corticoid release. Am. J. Physiol. 218:1632-1641. 
43. Smythe, G. A., J. E. Bradshaw, and R. F. Vining. 1983. Hypothalamic monoamine control of stress-induced adrenocorticotropin release in the rat. Endocrinology. 113:1062-1071.

44. Andersson, K., L, F. Agnati, K. Fuxe, P. Eneroth, A. Harfstrand, and F. Benfenati. 1984. Corticotropin-releasing factor increases noradrenaline turnover in the median eminence and reduces noradrenaline turnover in the paraventricular region of the hypophysectomized rat. Acta Physiol. Scand. 120:621-624.

45. Szafarczyk, A., G. Alonso, G. Ixart, F. Malaval, and I. Assenmacher. 1985. Diurnal-stimulated and stress-induced ACTH release in rats is mediated by ventral noradrenergic bundle. Am. J. Physiol. 249:E219-E226.

46. Szafarczyk, A., F. Malaval, A. Laurent, R. Gibaud, and I. Assenmacher. 1987. Further evidence for a central stimulatory action of catecholamines on adrenocorticotropin release in the rat. Endocrinology. 121:883-892.

47. Guillaume, V., B. Conte-Devolx, A. Szafarczyk, F. Malaval, N. Pares-Herbute, M. Grino, G. Alonso, I. Assenmacher, and C. Olivier. 1987. The corticotropin-releasing factor release in rat hypophysial portal blood is mediated by brain catecholamines. Neuroendocrinology. 46:143-146.

48. Plotsky, P. M. 1987. Facilitation of immunoreactive corticotropin-releasing factor secretion into the hypophysial portal circulation after activation of catecholaminergic pathways or central norepinephrine injection. Endocrinology. 121:924-930.

49. Damluji, S. A., L. Perry, S. Tormlin, P. Bouloux, A. Grossman, L. H. Rees, and G. M. Besser. 1987. Alpha-adrenergic stimulation of corticotropin secretion by a specific central mechanism in man. Neuroendocrinology. 45:68-76.

50. Greenwood, F. C., W. N. Hunter, and J. S. Glover. 1962. The preparation of ${ }^{131} I$ labelled human growth hormone of high specific radioactivity. Biochem. J. 89:114-123.

51. Duncan, D. B. 1955. Multiple range and multiple F tests. Biometrics. 11:1-42.

52. Zar, J. H. 1984. Biostatistical Analysis. Prentice-Hall, Inc., Englewood Cliffs, NJ. 718 pp.
53. Calogero, A. E., W. T. Gallucci, R. Bernardini, C. Saoutis, P. W. Gold, and G. P. Chrousos. 1988. Effect of cholinergic agonists and antagonists on rat hypothalamic corticotropin releasing hormone secretion in vitro. Neuroendocrinology. 47:303-308.

54. Svensson, T. H., B. S. Bunney, and G. K. Aghajanian. 1975. Inhibition of both noradrenergic and serotonergic neurons in brain by the $\alpha$-adrenergic agonist clonidine. Brain Res. 92:291-306.

55. Aghajanian, G. K., J. M. Cedarbaum, and R. Y. Wong. 1977. Evidence for norepinephrine mediated collateral inhibition of locus coeruleus neurons. Brain Res. 136:570-577.

56. Svensson, T. H., and T. Usdin. 1978. Feedback inhibition of brain noradrenaline neurons by tricyclic antidepressant: $\alpha$-receptor mediation. Science (Wash. DC). 202:1089-1091.

57. Jacobs, B. L. 1986. Single unit activity of locus coeruleus neurons in behaving animals. Prog. Neurobiol. (Oxford). 27:183-194.

58. Antoni, F. A. 1986. Hypothalamic control of adrenocorticotropin secretion: advances since the discovery of 41-residue corticotropin-releasing factor. Endocr. Rev. 7:351-378.

59. Herkenham, M. 1987. Mismatches between neurotransmitter and receptor localizations in brain: observations and implications. Neuroscience. 23:1-38.

60. Calogero, A. E., W. T. Gallucci, P. W. Gold, and G. P. Chrousos. 1988. Multiple feedback regulatory loops upon rat hypothalamic corticotropin-releasing hormone secretion. Potential clinical implications. J. Clin. Invest. 82:767-774.

61. Valentino, R. J., S. L. Foote, and G. Aston-Jones. 1983. Corticotropin-releasing factor activates noradrenergic neurons of the locus coeruleus. Brain Res. 270:363-367.

62. Valentino, R. J., and S. L. Foote. 1987. Corticotropin-releasing factor disrupts sensory responses of brain noradrenergic neurons. Neuroendocrinology. 45:28-36.

63. Gold, P. W., F. K. Goodwin, and G. P. Chrousos. 1988. Clini$\mathrm{cal}$ and biochemical manifestations in depression: relation to the neurobiology of stress. $N$. Engl. J. Med. In press. 